\title{
A World I Don't Inhabit: Disquiet and Identity In Second Life and Facebook
}

\author{
Stuart Boon, Christine Sinclair \\ CAPLE, University of Strathclyde,stuart.boon@strath.ac.uk, christine.sinclair@strath.ac.uk
}

\begin{abstract}
The authors use their own experiences of social software to argue for the need for caution in its uses in education. They particularly draw attention to difficulties in engagement, the effects on identity, an emphasis on superficial issues, lack of coherence, and problems with authenticity and trust. While Facebook and Second Life appear to have potential for educational applications, the disquiet associated with them may need to be taken into account: and this can affect both students and their teachers. One of the authors is a student on an online course and extracts from her blogs and journal capture the emotional and psychological effects of engaging in these new worlds at the time it happened. Both authors have noticed changes in their responses over time and point to the need for integration and coherence for "virtual identities" to play an appropriate part in higher education. The paper raises more questions than it answers and suggests that there is an urgent need to theorise online identity, roles of academics and students and codes of practice in such environments. If social software has a serious place in academic life, these issues will need to be widely debated.
\end{abstract}

\section{Keywords}

Social software, Identity, Disquiet

\section{Introduction}

Much of the rhetoric around online digital spaces understandably celebrates the potential of new environments such as Second Life and Facebook. These are spaces where human beings can creatively expand their personalities, activities, and opportunities for learning and communication. Higher Education has begun to focus excitedly on the benefits of these immersive spaces, seeing potential in new levels of engagement, increased interactivity and novel experiences that go well beyond what is currently possible in the classroom. But beyond all the hype, many users report feelings of discomfort and disquiet in these new domains. It is not just the well-publicised dark side of these spaces (e.g. see Borin, 2002) or mere unfamiliarity with hardware or software; this is a new kind of experience, a new metaphor, a new world in which to re/create ourselves, re/imagine our relationships to others, and re/evaluate the real and the unreal. While some meet these new opportunities with unbridled optimism and enthusiasm, others experience very real dissonance and doubt. This difference in experience is currently under-theorised and not very well understood. This paper seeks to outline the contexts in which disquieting and disturbing issues arise and make some hypotheses about their causes and implications.

In this paper we explore the experiences of the two authors in visiting these networked spaces and the disquiet encountered there. We focus specifically on our interactions with the social software application Facebook and the online simulation Second Life. We identify the extent to which our own experiences of the environments coincide with each other and with observations from the associated literature and the more anecdotal reports in the popular press. In doing this, we investigate the ontological status of avatars, profiles and other representations of a real life person and question how those constructed virtual identities impact the educational potential of the environments. We are keen to discover how the act of creating and "inhabiting" digital selves within virtual spaces affects not only what a student learns in these new spaces but also what a student may become in the process of engagement. 
One of us is undertaking a postgraduate course online and is observing her own responses to the language and activities she encounters. Extracts from her blogs and personal journal show a reluctance to engage with Second Life and Facebook combined with a curiosity and fascination about both of them. This has been a puzzle throughout the course and some examples from her blogs are used to illustrate our key points, showing the discomfort as recorded at the time it happened.

It should be said from the outset that neither of the authors is in any way averse to the use of information technology or to the application of that technology to pedagogy in Higher Education; in fact, quite the opposite is true, which makes our experiences of disquiet and dissonance even more profound, troubling and worthy of investigation. Nor are we completely against putting students in uncomfortable situations, as this can foster reflection and deep approaches to learning (Biggs, 2003): we just want to sound a few warning notes about it. We shall look at first at Facebook and then at Second Life in relation to our own experience as temporary inhabitants of these new social environments and in consideration of commentary from both the critical literature and more popular sources.

\section{Facebook}

The recent success of the social software application Facebook is nothing short of amazing and some of that success can surely be attributed to its integration with e-mail and its simple, familiar and attractive interface. For many of us, our first experiences with Facebook are initiated through our home or work e-mail accounts. As such, the application's reach is global and its method is viral: you might not be looking for Facebook, but Facebook is likely looking for you. One of us was reached this way, responding to repeated requests from friends; the other was asked to join as part of an academic course. This has prompted us to think about cohorts. If the decision to join stems from (a) peer pressure from a cohort of school students or (b) an attempt by academics to create an online cohort by encouraging participation, the result may be a new process of socialisation that academic cohorts have not experienced before. Within Facebook, in a relatively short amount of time, anyone can put much of their personal and professional life on display for others to peruse. It is at this point that some people begin to see questions forming, to feel a tug or two of nagging doubt.

I'm not sure that I like Facebook though and I've just realised it's because when we're meeting people we usually just release such details in small packets, when they're relevant or appropriate. And sometimes with other people it's good when they have matching characteristics; equally, sometimes, it's good when they don't. I've always wondered how the dating agencies take that into account.

Extract from student blog, shortly after joining Facebook when requested to by module leader

On the course identified in the extract, it is not compulsory to join or engage in Facebook though it is strongly encouraged. The fact that it brought to mind a dating agency to this middle-aged student perhaps suggests some of the identity issues that might arise. There was a question about whether this was appropriate for what we are supposed to be doing, especially in relation to the seductive and potentially time-consuming nature of the medium.

Others, perhaps even the majority of users, will not experience this questioning, even uncomfortable, feeling and will gladly engage in the process of creating and defining new digital selves. They will think little of re/presenting themselves via hypermedia: choosing what to reveal and what to withold, creating narratives that define 'who' they 'are' online, and providing digital photos and other artefacts which add verisimilitude like so much ocular proof. They may not feel the concern of others in putting so much of themselves and their lives onto the Internet, but feel greater concern in projecting a positive, attractive and even profitable vision of themselves - a face worthy of Facebook.

There are a number of reasons to question the appropriateness of Facebook as a cohort enhancer, though: it has been recorded that young students do not like teachers or parents in "their" space, especially if they are making embarrassing attempts to be cool; there is little control over what your 'friends' send you; the zany comment or picture that seemed like a good idea at the time later feels a bit frivolous, but the tracking system in Facebook makes you draw attention to it if you take it away. 
There may just be too much information about a teacher or a student - the loss of appropriate distance is part of the discomfort (e.g., see Hewitt and Forte, 2006; Mazer, 2006).

Responses to Facebook may go through various phases and the reluctance to release information about identity can ease over time as in the extract below shows:

My discomfort with Facebook has all sorts of sources - reluctance to create simple lists of preferences, dislike of the word "poke", not being photogenic (which I don't take as meaning the same as not being good-looking - though that may come into it as well!) I like things about Facebook too - I welcome its playfulness, its immediacy, its sense of belonging and for these reason will possibly join in more when I feel ready.

Extract from student blog, a few weeks into the module

Is a student who is persuaded to join in before she feels ready any more disadvantaged than when encouraged to engage in group work in a face-to-face class? Our answer is that we are not sure at this stage, but we do know that the situation is not the same.

Facebook allows its users, within its own predefined limits, to create a digital self that is at once fluid, protean, amorphous, and temporary. People alter their profiles daily, even hourly. Almost every piece of information is open to manipulation. This can make Facebook a marvellously creative environment, offering a number of novel opportunities. Seeing all this inherent possibility then, why should it make some of us so uncomfortable?

The answers to this question might not be found in the processes and subroutines of a piece of social software, but in our own human natures and our complex relationships with personal identity (Perry, 2002; Wood \& Smith, 2005) For example, questions of privacy and accessibility to information are inevitable in such an environment, even in a small network and using all the controls provided by Facebook itself. For some people, the issue of privacy alone can lead to considerable confusion and dismay concerning what information to divulge and what impact that exposure might have. Who will see this information? What will they think of me? How might that information be used? These questions are likely to result in some simply deleting their profiles and turning their backs on Facebook. For those who do come to terms with the privacy implications, the next and both more interesting and challenging question is how do we re/present ourselves in the digital world or 'Who am I?' in cyberspace (Suler 2002) - a question that could be asked not just by students but also by their lecturers (Ewins, 2005).

Cultural pressure, social mores and individual desire - or a mixture thereof - will lead most, if not all, of us to create idealisitic virtual re/presentations of our real world selves in Facebook. Having the ability to gently massage away the years, to add a little virtual muscle or brainpower, or appropriate the wit of Oscar Wilde or the eloquence of William Shakespeare, there are clearly few among us who can resist the temptation to act upon such impulses. Facebook profiles and Second Life avatars all contain an element of performativity in their makeup. Our life on the screen (Turkle, 1995) embodies, to one extent or another, a life on the stage, albeit a digital one. The selves we re/create on Facebook are inevitably part us - re-creating ourselves in digital form - and, again to one extent or another, part who we'd like to be - the creation of something new, perhaps better, but ultimately 'other.'

Again, some people will show little concern, if indeed any awareness of, this projection of identity with its performative aspects, but for others this awareness can be a point of surprising consternation and disquiet. This virtuality can be very much at odds with the reality we inhabit and people can begin to see their digital selves as superficial, artificial or even fraudulent. The very structure and functioning of Facebook seems to encourage superficiality, embracing the novel and the popular over the meaningful and complex. From hour to hour, day to day, users are inundated with notifications and requests from other users, user groups, applications, and Facebook itself asking for confirmations, installations, pokes, comments, and status updates. Games, polls, shared multimedia and a seemingly endless proliferation of associated applets provide enough empty content and low-level interactivity that some users can all but live on Facebook. Indeed on some profiles, it is worryingly difficult to 'find' the person in among all the digital artifice. 
The multiplicity and fluidity of identity within Facebook in itself can be a challenge to our real world cultural frameworks of truth and trust. Facebook user profiles are obvious constructs: there is truth in them, but invariably artifice as well. Thus, to some, these digital selves become fractured, confused reflections of a person, never wholly unreal, but never wholly real either - a seeming half truth. Disquiet arises then in having to ask the questions how much truth exists in a profile and how much trust should one attribute to the individual behind it? Even real world friendships can be confused or diminished by interactions with a digital self which seems to contradict the known real world self. The intermingling warp and weft of the real and the unreal can lead some to increased feelings of distrust and isolation.

Within the confines of Facebook, it is difficult to escape these doubts over individual authenticity and validity. The application itself is little more than a combination of e-mail and database programmes and has neither the functionality nor capability to ensure proper authenticity of identity. Even the most determined individual would find themselves frustrated when attempting to validate the 'realness' of their own or someone else's identity on Facebook. Digital selves invariably lack the solidity and verifiability of the real, particularly as they are both literally and figuratively 'unreal.' To say this might seem like stating the obvious, but what might not be obvious is the emotional effect that this realisation can have on the entire virtual experience. It can result in dissonance, sometimes even complete disconnection, between real-world experiences and experiences within a digital world or environment (e.g., see Land \& Bayne, 2006). Facebook does little to overcome this problem and, as we will see, Second Life does even less. In both cases, the onus is on the individual to break down or correct this dissonance.

When the authors of this paper discussed our own images on Facebook, we discovered that we had different reasons for presenting ourselves in the ways that we have done. One difference relates to the image of the self that can be accessed by anyone at all: should it be a 'real' photo or an avatar or other representation, such as a flower? For some people, photographs are reserved just for known people to see; it feels personal. On the online course, this topic also came up: some students did not use personal photos at all, some used long-shots, others wanted close ups to show themselves as approachable:

\begin{abstract}
I hate most photos of me - the one I sent in for my student card reminds me of the iconic picture of the British murderer Myra Hindley. But this one was taken by someone who knew what he was doing and it's the only one I've used online so far. Like R's, it does have additional meanings because of the time it was taken - and these are very positive.

A few weeks ago, I realised just how powerful avatars could be when I saw one that a friend had put on Facebook that seemed to capture her better than a photo. I thought - these are aspects of herself that she wants to display. Then I bumped into her one day and she told me that the avatar was out of date - it was four years old. I also realised that someone else had produced it for her. That made me reassess my view that it was how she wanted to present herself; it was actually how she did present herself to the rest of us.
\end{abstract}

Extract from a group blog, during discussion on presentation of self

The realisation that all profiles, to one extent or another, are 'unreal' can lead to further disquiet. Relationships with, and communication between, digital selves is therefore fraught with questions. The ambivalence of identity leads ultimately to low, even artificial, levels on engagement. When first engaging with Facebook, the level of interactivity may be quite high as people connect and re-connect with friends and colleagues, some of whom may have become quite distant. The game of connection sustains most users for a short period; however, once the many connections between digital selves within Facebook are made, the level of interactivity and engagement may fall off dramatically and the connections created appear weak or hollow compared to connections maintained in the real world.

Indeed, Facebook seems to encourage us to create essentially false communities of superficial relationships. That is not to say that the medium is never used to maintain strong communities of meaningful relationships, but rather that these seem to be in the minority. Instead, the groups and communities of Facebook show the same predilection for the new, the popular and the entertaining we noted earlier. Even as new users are trying to find a place and position for their digital selves, they may feel simultaneously impositioned as their e-mail inboxes and Facebook homepages fill up with 
invitations to groups and application requests from other users. The success of many groups and applications on Facebook appears to be achieved largely through social pressure and, thus, 'community' can seem disturbingly similar to conformism and forced community.

Superficial relationships are further supported by a system of communication within Facebook that prefers empty, phatic connection over high-content, meaningful communication. The poke function is a perfect example of this preference. In poking, Facebook gives us a way to say nothing while suggesting a relation or connection — a hollow verse saying 'I know/connect to you.' Pokes are even given pride of place on the Facebook homepage while the e-mail facility is tucked away behind a tab. The limited functionality and small form-boxes provided in the Inbox also seem to suggest that this is not the best way to communicate on Facebook. One-to-many forms of communication, such as status updates or a profile's Wall, are preferred over the one-to-one. Facebook seems to be saying, 'Why talk to one person when you can broadcast yourself to a wide audience?' But how many of us would care to broadcast something personally meaningful in this manner? And, if we are students, what are we actually saying to our teachers, our cohorts and our future employers?

I am coming self-conscious. It started with Facebook \& Second Life and continues there. It's also present in the group blog more than it was in the private blogs... Being self-conscious means that you wonder if you should do something differently so that people have a better opinion of you. Then you-I- - wonder if that means I'm so vain that I'll try to put on a public persona. But surely the real me will leak out.

Extract from student's personal journal during second year of course

When we have created several faces and lives for ourselves, we may even start to wonder which one is real.

\section{Second Life}

As an online simulation, Second Life adds greater complexity and increased artifice to the issues we've already raised in our discussion of Facebook. Greater complexity occurs in both form and function and, while we focus on questions of identity and disquiet here, there remain very real questions about the usability and accessibility of Second Life, particularly as it might be used en masse in Higher Education. Creating a digital self in Second Life is altogether a more sophisticated and involved process. Just getting into the world can be a problem:

The course is certainly enlightening about student experiences, including some I haven't had before but know that others have. The main one currently is being excluded from a classroom in SL because of where I come from (technically). Money will sort this out eventually ... but having spent some money [on computer memory] at a time when it's potentially a bit tight... I was frustrated that I still haven't solved the problem... For me, being excluded from SL yesterday gives me an additional insight into the emotional effects of exclusion - mainly frustration, but also some feeling of loss and inadequacy.

Extract from student's blog the day after the first course tutorial in Second Life

From registration through to immersion, Second Life guides the user through a series of steps that culminate in the creation of an animated, three-dimensional avatar: the digital expression of self in the expansive digital world. As with Facebook, for some this act of creation will be an enjoyable, even liberating experience wherein the imagination can run riot and every fancy can be entertained in the construction and elaboration of something that is truly 'other.' However, not all will find the experience so positive. From the outset, Second Life necessitates a commitment to the unreal, going so far as to make it impossible for users to use their own names. It is clear that there is an element of play involved here, as users can invest their avatars with some surprisingly inventive names, such as Wilde Cunningham or Archimedies Shostakovich, and perhaps there is some usefulness in providing a modicum of anonymity; however, for some this stripping of real identity can be both frustrating and unsettling. In Facebook, your name provides the strongest link between your digital and real world selves; in Second Life, that link is the first thing to be removed. It provides a very real disconnect from the real world. 
The creation of an avatar can lead to further disquiet in that it effects a symbolic disembodiment (Dreyfus, 2001). Expanding on the two-dimensional fabrication of digital self in Facebook, Second Life requires that we simulate our selves in a full three dimensions. Linden Labs, the developers of Second Life, have gone to great lengths to allow for avatars of all shapes and sizes to be manufactured. Users can attempt to replicate their real world bodies digitally, or they can create extravagant, idealised bodies, or go further still and inhabit animal or robot bodies. Within Second Life, identity as transmitted through an avatar is utterly variable: you can change colour, shape, gender, and even species with a few clicks of a mouse button. The avatar is artificiality writ large, a fabrication and, in some cases, a digital form devoid of personal significance.

There was a lot of talk about appearance [during a tutorial in Second Life] and I realised how little I bother with appearance most of the time. However, I do have some concerns in RL [shorthand for 'real life'] - hair colour (i.e. not grey) and facial expression - wanting to be approachable and friendly. I particularly respond to facial expression in others (and I'm not bothered about other people's hair colour, though might notice something stunning). These concerns seem to be carried over into SL and I'm not sure if I can get the facial expression right. Yet it was clear that it was overall impression that affected others more. This is a potential difference between SL and RL - or perhaps only for me. It's something I'll look out for-how important is facial expression going to be for me?

Extract from student blog after first major Second Life session (i.e. interacting with others)

Make no mistake, this artifice is part of Second Life's appeal, but it is also something of a problem for many users. Even within our own minds the awareness of, and friction between, who we are in real life and who we should be, might be, or may be compelled to be on screen can be disquieting. Who in fact do I want to be? Look ahead to interacting with other avatars in the simulated environment and the problem of identity is compounded further. As with Facebook, we again run into difficulties in determining trust and truth. In fact, within Second Life, it can be nearly impossible to assess the real identity and/or authenticity of another user. Taking this into account, who then can you trust? And what information can be believed or taken as true or authentic? It is entirely possible to create multiple identities on Second Life and to share accounts and identities, though the latter is formally discouraged. All this being true, the arising dilemma then is: how can you ever be certain of the identity of another in Second Life? The answer - itself a point of considerable disquiet - is that there virtually is no way to acertain the authenticity of another user through their avatar. So who indeed can you trust and what can you believe in this environment?

The simulated environment itself and the movement of avatars throughout it again highlights the performativity of these digital spaces. Even more than Facebook, the world of Second Life is a virtual stage (Guest, 2007). Filled with artifice and enthralling spectacle, the simulated environment is both highly immersive and highly seductive; it is a world that draws people in, offering them experiences that could never be had in the real world, removed from risk and consequence. In Second Life, we can fly, teleport from island to island, we can walk on lava and in doing so we never need worry about coming to harm or dying. The simulation is like a digital limbo in which our digital selves entertain themselves throughout eternity - providing fees are paid up on time. The 'freedom to act' offered by Linden Labs in Second Life may well be a dual-edged sword: freedom to create wonders and to do and experience the impossible, but freedom also to disrupt, destroy or inspire hate.

Second Life, like every successful massively multiplayer online role-playing game (MMPORG), provides a seductive combination of the real and the unreal: real enough to allow us to interact with it, to understand simple laws like gravity or cause and effect, but unreal and fantastic enough to entice us towards exploration and experimentation (Castronova, 2005). This together with the ability to create an identity and to progress or better ourselves in some fashion is a heady mix. For the escapist or the world-weary, it is a dream come true and its effect can be disturbingly drug-like. For some Second Life provides just that: a second life, an escape from the real world and all its messiness, its problems and limitations. More than Facebook ever could, Second Life removes the user from the real world.

I had an unusual effect on Friday when I left the screen for a moment to look for the reading... In the few second that I left the screen, someone spoke to me in SL and I had to get back quickly. I had an interesting sensation of jumping between worlds. 
Digital spaces cannot yet contain the messy complexities of the real and most would not wish them to. A proportion of people using Second Life or any MMPORG are doing so-wittingly or unwittinglyto avoid real life issues and situations. The same could be said of television, of going to a theatre or reading a book, but the difference is the amount of time and mental effort involved. Maintaining and progressing a virtual identity inside Second Life require considerable more effort and time than does sitting in a movie theatre, watching a television screen, or reading a book. It is entirely possible to find every waking moment devoured by the thoughts and activities required in leading 'two' lives.

A situation exists wherein some users might be served well by this means of escape, but still others will not benefit, unless perhaps momentarily, from such escape and even be unsettled by it:

If I alter my conceptions about the way things happen with a game or virtual environment, is this as real as altering them within a 'real world' experience?

So, is there a dark side that might be outed by games and SL and that can't be put back in once it's let out?

Two extracts, several days apart, from student's blog during module on computer gaming

The impact then of having a virtual identity in Second Life and of inhabiting these digital spaces, can have a tangible impact on our real lives. While so many people might be attracted to the immersive escape from reality offered by these new social technologies, few of us seem to realise — at least initially_what toll this escape can have on our lives outside the screen.

\section{Educational Uses/Limitations of Facebook and Second Life}

As of the time of this writing, universities around the globe are buying up property in Second Life and asking how they might harness the popularity and functionality of Facebook. Our own institution has just purchased an island and discussion on the uses of social software are becoming more and more common. Our own analysis above already indicates that we can see that Facebook and Second Life will have some, potentially quite exciting, educational uses; however, we also note that those uses might not be without challenges or costs to students, academics, and institutions. It is clear that social software might be put to good use in some contexts and be less useful in others.

The potential uses of Facebook are perhaps more limited than those of Second Life, but the connectivity of Facebook mixed with its ability for integrated multimedia, polling, and asynchronous chat could be put to use in an academic setting. Second Life's immersive virtual world is open to even greater possibilities with the ability to simulate situations and events from the sub-atomic to the global - it can truly offer learners experiences that could not be had in the real world, whether they purely theoretical or involve great risk. But it is exactly this kind of removal or distancing from real world experience that seems to likewise result in some potential limitations and to feelings of disquiet and discomfort.

Neither Facebook nor Second Life was developed as an educational tool and students' experiences in using these technologies may vary greatly. The omnipresent artificiality of identity within these spaces and the concommitant challenges to frameworks of trust and truth may leave some students feeling distanced, isolated, or even disconnected. As the current applications are both designed for and prefer the novel, popular and phatic over the meaningful, factual and content-driven, academics might find themselves faced with questionable levels of engagement and difficulty in achieving meaningful, deep learning for their students. And as we have discussed, taking experience out of the real world, divorcing it from risk and real world consequence, may have the effect of subsequently diminishing or altering its real world significance. Some might go so far as to question whether inhabiting digital selves in online social spaces could ever provide 'real experiences' that reinforce 'real' learning?

More practically, the management and security of classes run in either of these environments could be very big challenges to busy academics, as could dealing with the expectations and needs of a diverse 
student population suddenly made digital. Will academics have to deal with a doubling of student numbers as they take on virtual identities? What is clear is that a great deal more research needs to be done on aspects such as how these social spaces might function as educational spaces and how inhabiting these spaces will provide challenges and opportunities for future learners.

\section{Real World Implications and Conclusions}

Our conclusions are not as negative as the title of this paper might suggest. We have found that asking these questions about Facebook and Second life has led us to raise issues about our offline practices too-placing our real world beliefs "within brackets" in a revealing way (Evans, 2000). This is no bad thing and ought to be encouraged in students as well.

In particular, we have shown that the discomfort draws attention to unexamined aspects of presentation of self to others, academic and social assumptions and conceptualisations, and even our moral stances. While some of these observations are common to education in general, what is new is the disjunction between various different types of experience which are simultaneously demanding our intellectual attention. As we tune in to one aspect of our experience, the seductive pull of some its more trivial cousins may make it more difficult for us to make any meaningful use of it. Critical engagement and depth of study may give way to identity management and loss of values.

Some of the unresolved issues that students and their teachers may need to address are:

1. Who is taking this degree - a particular person, an idealised version of that person, a social group?

2. How can the integration of different aspects of identity be most appropriately managed - if indeed it should be - and whose responsibility is this?

3. Do we need different codes of academic behaviour to deal with shifting identities?

In particular, our concern is that we ourselves and our colleagues may not be well-equipped to deal with unanticipated emotional and personal responses arising from these questions.

\section{References}

Biggs, John B. (2003) Teaching for quality learning at university. Second Edition. Buckingham: SRHE/Open University Press.

Borin, E (2002) 'Report: Net Not Getting Any Safer.' Wired. 10.09.02. [Accessed 10 January 2008] http://www.wired.com/techbiz/media/news/2002/10/55581

Castronova, E. (2005) Synthetic Worlds: The Business and Culture of Online Games. Chicago: University of Chicago Press.

Dreyfus, Hubert L. (2001) On the Internet. London: Routledge.

Ewins, R. (2005) 'Who are You? Weblogs and Academic Identity', E-Learning, Volume 2, Number 4, pp. $368-377$.

Guest, T. (2007) Second Lives: A Journey Through Virtual Worlds. London: Hutchison.

Hewitt, A. and Forte, A. (2006) 'Crossing Boundaries: Identity Management and Student/Faculty Relationships on the Facebook' CSCW '06 November 4-8 Banff, Alberta, Canada [Accessed 10 January 2008] http://www.cetis.ac.uk/members/scott/blogview? entry=20070426014059

Land, R. and Bayne, S. (2005) 'Issues in cyberspace education' Problem-based Learning Online. Savin-Baden and Wilkie, eds. Maidenhead: Open Universit Press.

Mazer, J. (2006) 'I'll see you on "Facebook": the Effects of Computer-Mediated Teacher SelfDisclosure on Student Motivation, Affective Learning, and Classroom Climate 1

Perry, J. (2002) Identity, Personal Identity, and the Self. Indianapolis: Hackett.

Suler, J.R. (2002) Identity Management in Cyberspace. Journal of Applied Psychoanalytic Studies, 4, 455-460

Turkle, S. (1995) Life on the Screen. London: Weidenfeld and Nicholson.

Wood, A. and Smith, M. (2005) Online Communication: Linking Technology, Identity \& Culture. Mahwah: Lawrence Erlbaum. 